\author{
Edoardo Baglivo \\ André Dosso \\ Constantin Pournaras
}

\section{Thrombus and branch retinal vein occlusion}

hyperlipidaemia. After 2 months, fundus examination revealed disappearance of the intravascular thrombus, resolution of the macular edema and improvement of the visual acuity. Certain physiological characteristics of the retinal circulation associated with hyperlipidaemia and systemic hypertension appear to favour thrombus forma- tion.

fax +41-22-382.83.82;

e-mail

baglivo-edoardo@diogenes.hcuge.ch

\begin{abstract}
Branch retinal vein occlusion (BRVO) is often associated with arteriosclerosis. Typically the occlusion occurs at an arteriodeveloped a BRVO. Funduscopy and fluorescein angiography sug gested an intravascular thrombus as the cause of the occlusion. The investigations performed were positive for systemic hypertension and
\end{abstract}

\section{Introduction}

Acute branch retinal vein occlusion (BRVO) presents a characteristic clinical picture with flame-shaped and dot and blot haemorrhages, soft and hard exudates, retinal oedema and a dilated vein in a segmental distribution. The occlusion invariably appears to occur at an arteriovenous (AV) crossing point $[9,10]$; thickening of the arterial wall and the presence of a common adventitia of the artery and the vein at $\mathrm{AV}$ crossings seem to play a role in the pathogenesis of the BRVO $[10,16]$.

The major risk factor is arteriosclerosis (age, systemic hypertension, diabetes mellitus, smoking, hyperlipidaemia). Other predisposing factors, such as blood dyscrasias, lympho-proliferative disorders, periphlebitis (sarcoidosis, Behcet's disease, retinal infections), hyperopia and open-angle glaucoma have been described [1-3, $12,15,17]$.

We report here the case of a 53-year-old man who developed an occlusion of the superior temporal vein. Funduscopy and fluorescein angiography of the occlusion suggested the presence of an intravascular thrombus.

\section{Case report}

A 53-year-old previously healthy man presented in December 1992 with a 2-day history of sudden decrease of visual acuity in his left eye.

On examination, the patient's visual acuity for distance was $10 / 10$ with -0.75 in the right eye, and the best corrected visual acuity in the left eye was $5 / 10$. Intraocular pressure was $10 \mathrm{mmHg}$ in both eyes. Results of slit-lamp examination were normal in each eye. Fundus examination of the right eye showed minimal arteriosclerosis. In the left eye, a superotemporal BRVO was noted. Whitish intravascular material was observed in a segment of the occluded vein at two dise diameters from the optic disc (Fig. 1). The fluorescein angiography showed staining of the venous wall at distance of the proximal end of the intravascular material. The hyperfluorescence was visible in the early arteriovenous phase and increased in the mid-phase of the angiogram. Distal to the intravascular material, the retinal venous circulation time was increased (Fig. 2a). At the site of the venous occlusion, the biomicroscopic and angiographic findings suggested that this material was an intravascular thrombus. Moreover, in this area, a breakdown of the blood-retinal barrier was observed (Fig. 2b).

We performed investigations for systemic hypertension, diabetes mellitus, hyperlipidaemia, connective tissue disorder, $\mathrm{Be}$ hcet's disease, sarcoidosis and blood dyscrasia. Results were positive for systemic hypertension and hyperlidaemia [HDL cholesterol $0.52 \mathrm{mmol} / \mathrm{l}(n=0.75-1.73)$; total cholesterol $8.03 \mathrm{mmol} / 1$ $(n=3.3-6.5) ;$ LDL cholesterol $4.81 \mathrm{mmol} / 1(n=$ less than 3.9); 

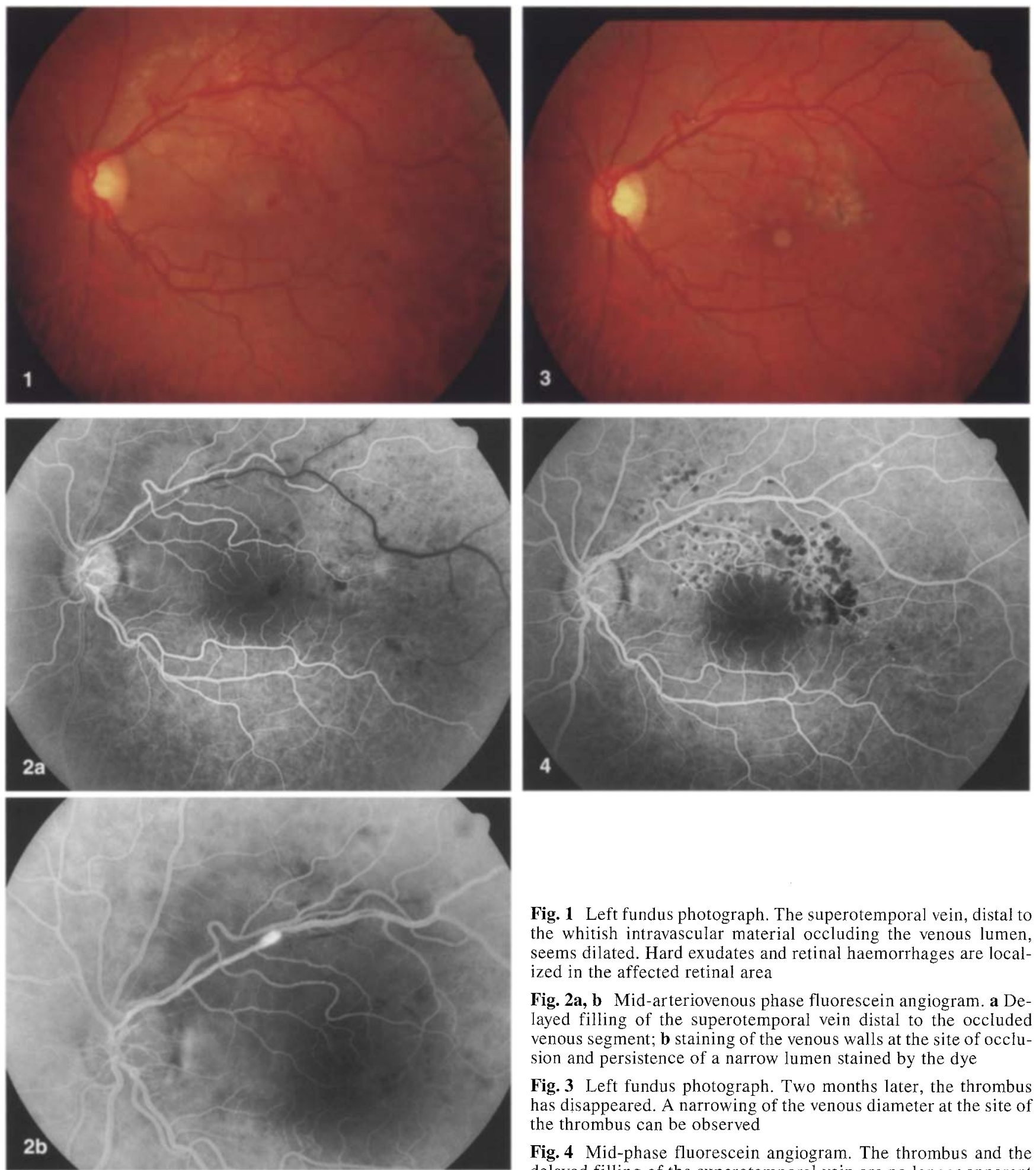

Fig. 1 Left fundus photograph. The superotemporal vein, distal to the whitish intravascular material occluding the venous lumen, seems dilated. Hard exudates and retinal haemorrhages are localized in the affected retinal area

Fig. 2a, b Mid-arteriovenous phase fluorescein angiogram. a Delayed filling of the superotemporal vein distal to the occluded venous segment; $\mathbf{b}$ staining of the venous walls at the site of occlusion and persistence of a narrow lumen stained by the dye

Fig. 3 Left fundus photograph. Two months later, the thrombus has disappeared. A narrowing of the venous diameter at the site of the thrombus can be observed

Fig. 4 Mid-phase fluorescein angiogram. The thrombus and the delayed filling of the superotemporal vein are no longer apparent

triglycerides $5.94 \mathrm{mmol} / 1(n=0.4-1.83)]$. We excluded secondary dyslipidaemia (hypothyroidism, diabetes, liver disease, renal disease). The patient did not drink alcohol, did not smoke and was neither obese nor a drug abuser.The systemic hypertension was treated with a calcium channel blocker and the dyslipidaemia with hypolipaemic medication (benzylfibrate), and we introduced salicylates $(100 \mathrm{mg} /$ day $)$. Dietary advice was given.

Two days later, the visual acuity of the left eye had worsened $(3 / 10)$ and retinal examination showed macular oedema. Due to the persistence of the macular oedema and the presence of is- 
chaemic areas, argon laser photocoagulation was performed 6 weeks after the diagnosis.

Two months later, the visual acuity of the left eye had improved $(10 / 10$ without correction). Fundus examination showed the disappearance of the whitish intravascular material and a resolution of the macular oedema (Fig. 3). Fluorescein angiography revealed reperfusion of the superior temporal vein (Fig. 4). Finally, the lipidogram and the patient's blood pressure were normal.

\section{Discussion}

Leber [14] was the first to suggest the importance of arteriosclerosis and the vulnerability of $\mathrm{AV}$ crossing sites in BRVO. Jensen observed that the artery lay anterior to the vein at the majority of BRVO crossing sites and postulated that the predilection of BRVO for the superotemporal quadrant was due to the greater number of crossings in this quadrant [11]. Others have recently confirmed Jensen's observation of a greater percentage of artery-anterior-to-vein crossings at the BRVO sites [7, 19] and of a greater number of crossings in the superotemporal quadrant [8]. A histopathologic study confirmed the importance of the $\mathrm{AV}$ crossing at the site of the BRVO [10]. In our case, we observed, at the site of the occlusion, an intravascular white material occluding the vessel's lumen without any sign of ocular inflammation. This material disappeared two months later.

These observations suggest that the cause of the occlusion was the presence of a thrombus. The follow-up was characterized by the disappearance of the thrombus and the recanalisation of the superotemporal vein. As risk factors, our patient had high blood pressure and hyperlidaemia. This seems to be the first clinical report of a BRVO caused by a thrombus.

Since the Framingham study [13], hyperlidaemia as an isolated predisposing factor furthering atherosclerosis in large vessels or an ischaemic arterial stroke has been well documented [18]. Nevertheless, few studies describe venous thrombosis occurring in association with dyslipidaemia [4].

Dodson and colleagues $[5,6]$ found a greater prevalence of hyperlipidaemia and hypercholesterolaemia in patients suffering from BRVO than in an age-matched group. They proposed that the increase of serum lipids may contribute to the aetiology of vein occlusion by altering plasma viscosity or affecting platelet function.

There is general agreement that the origin of BRVO includes both systemic factors such as hypertension and local anatomical factors such as AV crossings. However, the initial phenomenon causing such condition is still uncertain.

In our case, the mechanical factor was absent. Whether the thrombus formation was due to abnormal blood viscosity or alteration of platelet function, induced by hyperlipidaemia, could not be ascertained. However, it appears that hyperlipidaemia and hypertension may favour intravenous thrombus formation, and the treatment of risk factors seems to improve the natural course of the disease.

\section{References}

1. Appiah AP, Greenidge KC (1987) Factors associated with retinal vein occlusions in Hispanics. Ann Ophthalmol 19:307-309

2. Appiah AP, Trempe CL (1989) Differences in contributory factors among hemicentral, central, and branch retinal vein occlusions. Ophthalmology 96:364-366

3. Coscas G, Dhermy P (1978) Occlusions veineuses rétiniennes. (Société française $d^{\prime}$ ophtalmologie) Masson, Paris

4. De Gennes JL, Ozanne P, Turpin G (1982) Les accidents thrombo-emboliques veineux au cours des hyperlipidémies idiopathiques. Nouv Presse Med 23: 1777-1780

5. Dodson PM, Galton DJ, Winder AF (1981) Retinal vascular abnormalities in the hyperlipidaemias. Trans Ophthalmol Soc UK 101: 17-21
6. Dodson PM, Galton DJ, Hamilton AM, Blach RK (1982) Retinal vein occlusion and the prevalence of lipoprotein abnormalities. $\mathrm{Br} \mathrm{J}$ Ophthalmol 66: 161-164

7. Duker JS, Brown GC (1989) Anterior location of the crossing artery in branch retinal vein obstruction. Arch Ophthalmol 107:998-1000

8. Feist RM, Ticho BH, Shapiro MJ, Farber M (1992) Branch retinal vein occlusion and quadratic variation in arteriovenous crossings. Am J Ophthalmol 113:664-668

9. Finkelstein D (1989) Retinal branch vein occlusion. In: Ryan SJ, Schachat AP, Murphy RP, Patz A (eds) Medical retina, vol 2. Mosby, St Louis, pp $427-432$

10. Frangieh GT, Green R, BarraquerSomers E, Finkelstein D (1982) Histopathologic study of nine branch retinal vein occlusions. Arch Ophthalmol 100: 1132-1140
11. Jensen VA (1936) Clinical studies of tributary thrombosis in the central retinal vein. Acta Ophthalmol Suppl 10: 1-193

12. Johnston RL, Bruckner AJ, Steinmann W, Hoffmann ME, Holmes JH (1985) Risk factors of branch retinal vein occlusion. Arch Ophthalmol 103:18311832

13. Kannel WB, Castelli WP, Gordon T, et al (1971) Serum cholesterol, lipoproteins, and the risk of coronary heart disease. The Framingham study. Ann Intern Med 74: 1-11

14. Leber T (1877) Die Krankheite der Netzhaut und des Sehnerven. In: Graefe A, Saemisch T (eds) Handbuch der Gesammten Augenheilkunde: Pathologie und Therapie. Engelmann, Leipzig, pp 521-535 
15. McGrath MA, Wechsler F, Hunyor AB, Penny R (1978) Systemic factors contributory to retinal vein occlusion. Arch Intern Med 138:216-220

16. Orth DH, Patz A (1978) Retinal branch vein occlusion. Surv Ophthalmol 22: 357-376
17. Rath EZ, Frank RN, Shin DH, Kim C (1992) Risk factors for retinal vein occlusions. Ophthalmology 99:509514

18. The Lipid Research Clinics Coronary Primary Prevention Trial (1984) The lipid research clinics coronary primary prevention trial results. 1. Reduction in incidence of coronary heart disease. 2 . The relationship of reduction in incidence of coronary heart disease to cholesterol lowering. JAMA $251: 351-364$
19. Weinberg D, Dodwell DG, Fern SA (1990) Anatomy of arteriovenous crossings in branch retinal vein occlusion. Am J Ophthalmol 109:298-302 